\title{
RNA virome diversity and Wolbachia infection in individual Drosophila simulans flies
}

\author{
Ayda Susana Ortiz-Baez¹, Mang Shi', Ary A. Hoffmann² and Edward C. Holmes ${ }^{1, *}$
}

\begin{abstract}
The endosymbiont bacteria of the genus Wolbachia are associated with multiple mutualistic effects on insect biology, including nutritional and antiviral properties. Members of the genus Wolbachia naturally occur in fly species of the genus Drosophila, providing an operational model host for studying how virome composition may be affected by its presence. Drosophila simulans populations can carry a variety of strains of members of the genus Wolbachia, with the wAu strain associated with strong antiviral protection under experimental conditions. We used D. simulans sampled from the Perth Hills, Western Australia, to investigate the potential virus protective effect of the wAu strain of Wolbachia on individual wild-caught flies. Our data revealed no appreciable variation in virus composition and abundance between individuals infected or uninfected with Wolbachia associated with the presence or absence of wAu. However, it remains unclear whether wAu might affect viral infection and host survival by increasing tolerance rather than inducing complete resistance. These data also provide new insights into the natural virome diversity of $D$. simulans. Despite the small number of individuals sampled, we identified a repertoire of RNA viruses, including nora virus, galbut virus, thika virus and La Jolla virus, that have been identified in other species of the genus Drosophila. Chaq virus-like sequences associated with galbut virus were also detected. In addition, we identified five novel viruses from the families Reoviridae, Tombusviridae, Mitoviridae and Bunyaviridae. Overall, this study highlights the complex interaction between Wolbachia and RNA virus infections and provides a baseline description of the natural virome of $D$. simulans.
\end{abstract}

\section{DATA SUMMARY}

The authors confirm all supporting data, code and protocols have been provided within the article or through supplementary data files.

\section{INTRODUCTION}

The members of the alpha-proteobacterium genus Wolbachia (order Rickettsiales) are widespread endosymbionts of arthropods and nematodes (i.e. filarial and plant-parasitic nematodes) that can establish interactions with their hosts ranging from parasitic to mutualistic $[1,2]$. The genetic diversity of the genus Wolbachia is substantial and currently represented by 11 distinctive supergroups (denoted $\mathrm{A}-\mathrm{J}$ ), although the majority of strains of members of the genus Wolbachia belong to supergroups A and B [3] that are estimated to have diverged around 50 million years ago [4]. Although these bacteria are commonly found in reproductive tissues and the germline of their hosts, they have also been found in somatic tissues, such as the brain, salivary glands and gut [5-9], such that understanding infection dynamics in detail is not a trivial matter [7]. Members of the genus Wolbachia primarily spread

Received 26 April 2021; Accepted 01 July 2021; Published 27 October 2021

Author affiliations: 'Marie Bashir Institute for Infectious Diseases and Biosecurity, School of Life and Environmental Sciences and School of Medical Sciences, The University of Sydney, Sydney, New South Wales 2006, Australia; ${ }^{2}$ School of BioSciences, Bio21 Institute, University of Melbourne, Parkville, Victoria 3010, Australia.

*Correspondence: Edward C. Holmes, edward.holmes@sydney.edu.au

Keywords: evolution; Drosophila simulans; meta-transcriptomics; phylogeny; RNA virome; Wolbachia.

Abbreviations: cox1, cytochrome c oxidase I; DCV, drosophila C virus; DENV, dengue virus; DNA, deoxyribonucleic acid; F1, first filial generation; FHV, flock house virus; HRM, high-resolution melt assay; K-S, kolmogorov-smirnov; NCBI-nr, NCBI non-redundant; ORF, open reading frame; PBS, phosphate buffered saline; PCR, polymerase chain reaction; qPCR, quantitative PCR; RdRp, RNA-dependent RNA polymerase; RNA, ribonucleic acid; RNA-Seq, RNA sequencing; RPL32, ribosomal protein L32 gene; RPM, reads per million; rRNA, ribosomal RNA; RT, real-time PCR; SH-aLRT, Shimodaira-Hasegawa-like approximate likelihood ratio test; SRA, sequence read archive; UFboot, ultrafast bootstrap approximation; wsp, wolbachia surface protein; ZIKV, Zika virus.

The viral genome sequence data generated in this study have been deposited in the NCBI/GenBank database under the accession numbers MW976812-MW976882. Sequence reads are available at the public Sequence Read Archive (SRA) database under the BioProject accession PRJNA706433 (BioSample accessions: SAMN18132282-SAMN18132297).

One supplementary figure and four supplementary tables are available with the online version of this article. $001639 \odot 2021$ The Authors 
by vertical inheritance through transovarian transmission. However, the presence of members of the genus Wolbachia in a diverse range of host species indicates that horizontal transmission, probably through antagonistic interactions (i.e. herbivory, parasitism and predation), also contributes to the dissemination of the bacteria in nature $[4,10]$.

The occurrence of bacteria of the genus Wolbachia in insects is often associated with their ability to manipulate host reproductive mechanisms and induce a range of alterations, including parthenogenesis, feminization, cytoplasmic incompatibility and sex-ratio distortion [11]. Among these, cytoplasmic incompatibility is the most common phenotypic effect, and as such represents an appealing approach for vector population control. In this case, embryonic lethality is contingent on the infection status and the strain type harboured by males and females [2]. In addition, the study of Wolbachia-host interactions has revealed a variety of mutualistic effects on host biology $[1,12]$. For instance, in filarial nematodes and the parasitoid wasp Asobara tabida, the presence of some strains of members of the genus Wolbachia has been positively associated with developmental processes, fertility and host viability [12-14]. Furthermore, nutritional mutualism between members of the genus Wolbachia and the bedbug Cimex lectularius as well as Wolbachia-infected planthoppers, has been suggested as a means to explain B vitamin supplementation [15-17].

Arguably the most important outcome of Wolbachia infection in insects is its potential for virus-blocking, which also provides a basis for intervention strategies based on the control of arbovirus transmission. This seemingly antiviral effect of members of the genus Wolbachia has been well documented in some species of insects, including flies and mosquitoes. A striking example involves the transinfection of Aedes aegypti mosquitoes with a strain of the genus Wolbachia infecting Drosophila melanogaster $(w \mathrm{Mel})$. A. aegypti is the primary vector of a number of important arboviruses, including members of the species Dengue virus, Zika virus and Chikungunya virus, and the establishment of the wMel strain in wild mosquito populations represents a powerful and promising approach to decrease virus transmission $[18,19]$. Although the underlying mechanisms remain to be fully determined, it has been suggested that members of the genus Wolbachia can modify the host environment or boost basal immunity to viruses by pre-stimulating the immune response of their hosts [20]. Potential antiviral mechanisms affected by members of the genus Wolbachia include gene expression of the Toll pathway, RNA interference, and modification of the host oxidative environment that probably trigger an antiviral immune response and hence limit infection [20-22].

Unlike A. aegypti mosquitoes, members of the genus Wolbachia naturally occur in species of the genus Drosophila, providing a valuable model system to study Wolbachia-related virus protection $[23,24]$. Natural populations of species of the genus Drosophila can carry a diverse array of insectspecific viruses belonging to the families Picornaviridae, Dicistroviridae, Bunyaviridae, Reoviridae and Iflaviridae amongst others [25]. The co-occurrence of members of the genus Wolbachia in D. melanogaster has been associated with increased survival and different levels of resistance to laboratory viral infections in fly stocks under experimental conditions $[23,26]$. For example, Wolbachia-infected flies containing the dicistrovirus Drosophila C virus (DCV) showed a delay in mortality compared with Wolbachia-free flies [26]. In contrast, other studies found no or limited effect of members of the genus Wolbachia on viral protection, as well as on virus prevalence and abundance in field-collected flies $[25,27]$. Such contrasting data emphasize the need for further research efforts to characterize the effect of strains of the genus Wolbachia on virus composition in members of the genus Drosophila in nature.

Although the origin of $D$. simulans is thought to have been in East Africa or Madagascar, this species now has a cosmopolitan distribution [28]. In Australia, D. simulans has been recorded along both the east and west coasts as well as Tasmania, with the earliest record dating to 1956 [29]. Human mobility and human-mediated activities have been associated with the introduction and spread of both $D$. simulans and Wolbachia into Australia, where wild fly populations occur near human settlements, feeding and breeding on a variety of horticultural crops [30,31]. Several strains of members of the genus Wolbachia from supergroups A and B can naturally occur in populations of $D$. simulans (e.g. $w \mathrm{Au}, w \mathrm{Ri}, w \mathrm{Ha}$, $w \mathrm{Ma}$ and $w \mathrm{No}$ ) [32,33]. Of these, $w \mathrm{Au}$ is associated with strong antiviral protection against Flock House virus (FHV) (Nodaviridae) and DCV (Dicistroviridae) under experimental conditions [32]. The $w$ Au infection in Australia was one of the first infections by members of the genus Wolbachia identified as showing no cytoplasmic incompatibility, despite being widespread at a low to intermediate frequency [34]. wAu increased in frequency along the east coast of Australia until it was replaced by $w$ Ri that exhibits cytoplasmic incompatibility. However, unlike $w \mathrm{Au}, w \mathrm{Ri}$ has not yet reached the Australian west coast [30]. In this study, we used a meta-transcriptomic (i.e. RNA shotgun sequencing) approach to determine the virome diversity of individual field-collected $D$. simulans flies from Western Australia, and investigated how this virome diversity might be affected by the presence of the $w \mathrm{Au}$ strain of Wolbachia.

\section{METHODS}

\section{D. simulans collection and taxonomic identification}

Flies used for the virus work performed here were collected at Raeburn Orchards in the Perth Hills in Western Australia (latitude $-32.1036^{\circ}$, longitude $116.0695^{\circ}$ ), in July 2018 using banana bait. The frequency of members of the genus Wolbachia at two other locations in the area (Roleystone, latitude $-32.1396^{\circ}$, longitude $116.0701^{\circ}$; Cannington, latitude $-32.0243^{\circ}$, longitude $115.9363^{\circ}$ ) was also established with additional samples. Taxonomic identification to the species level was conducted on the basis of the morphology of reproductive traits of males and via DNA barcoding (cox1 gene marker). Field-collected flies were maintained at $19^{\circ} \mathrm{C}$ 
under standard laboratory conditions until F1 offspring were raised. Parental and $\mathrm{F} 1$ generations were then stored at $-80^{\circ} \mathrm{C}$ until molecular processing.

\section{Wolbachia detection}

Wolbachia infection of field females was determined using F1 offspring from each field female. Note that $w \mathrm{Au}$ is transmitted at $100 \%$ from field females to the F1 laboratory generation [34]. DNA extraction from heads was performed using Chelex 100 Resin (Bio-Rad Laboratories,) [35] as adapted y Shi et al. [27]. Screening of natural infection with members of the genus Wolbachia was conducted using a real-time PCR/ high-resolution melt assay (RT/HRM) and strain-specific primers targeting a $340 \mathrm{bp}$ region of the surface protein of Wolbachia (wsp) gene for $w \mathrm{Ri}$ and $w \mathrm{Au}$ strains. The assay was run following the protocol of Kriesner et al. [30]. In addition, reads were mapped to reference Wolbachia wsp gene sequences for $w \operatorname{Ri}(\mathrm{CP} 001391.1)$ and $w \mathrm{Au}(\mathrm{LK} 055284.1)$ with BBMap v.37.98 (minid=0.95) (available at https://sourceforge. net/projects/bbmap/).

\section{RNA extraction and meta-transcriptome sequencing}

We screened a total of 16 individual flies to assess the effect of infection with members of the genus Wolbachia on virome composition in $D$. simulans. Specimens were rinsed three times in RNA and DNA-free PBS solution (GIBCO). Total RNA from individual flies was extracted using the RNeasy Plus Mini Kit (Qiagen) following the manufacturer's instructions. RNA-seq libraries were constructed using a TruSeq total RNA Library Preparation Kit (Illumina). Host ribosomal depletion was performed using a Ribo-Zero Gold rRNA Removal Kit (Human/Mouse/Rat) (Illumina) and paired-end transcriptome sequencing was performed on the HiSeq2500 platform (Illumina). Libraries from Wolbachia-negative and -positive infected flies were run in two separate lanes.

\section{De novo meta-transcriptome assembly and viral genome annotation}

The overall quality assessment of reads was conducted in FastQC and Trimmomatic [36]. A de novo assembly of RNA-Seq data was performed using MEGAHIT v.1.1.3, with default parameters [37]. Assembled contigs were then annotated through comparisons against the NCBI nonredundant (NCBI-nr) database using DIAMOND v2.0.4 [38], with a cutoff e-value $<1 \mathrm{e}^{-05}$. To identify protein-encoding sequences, open reading frames (ORFs) were predicted in positive and reverse-complement strands, with a minimum length of $600 \mathrm{nt}$ between two stop codons using the GetOrf program (EMBOSS) [39]. Functional annotation was carried out using InterProScan v5.39-77.0 [40], and the HMMer software (http://hmmer.org/) was used to perform sequence-profile searches against the Pfam HMM database. To expand the de novo assembled contigs of known viruses, the reads were mapped against reference genomic sequences. Provisional virus names were derived from geographic locations in the Perth Hills, Western Australia.

\section{Estimates of viral abundance}

Viral abundance was assessed using the number of reads per million (RPM). This metric quantifies the number of reads per million mapped to a given contig assembly over the total number of reads. RPM values lower than $0.1 \%$ of the highest count for each virus across samples were presumed to be index-hopping artefacts and excluded from the remaining analyses [41]. To compare abundance levels, reads were mapped to reference ribosomal and mitochondrial genes from Wolbachia (16S and cox1) and D. simulans (rpl32 and cox1), as well as against all the RNA viruses identified in the annotation analyses. Mapping was performed using BBMap v.37.98 (minid=0.95) (available at https://sourceforge.net/ projects/bbmap/).

\section{Sequence alignment and phylogenetic analysis}

RNA viral sequences identified in $D$. simulans were compared with homologous reference sequences retrieved from the NCBI GenBank database and aligned with MAFF v7.450 (E-INS-I algorithm) [42]. Phylogenetic trees for these data were then inferred using sequences of the conserved RNAdependent RNA polymerase (RdRp) gene. To this end, both the best-fit model of amino acid substitution and phylogenetic relationships were estimated using the maximum likelihood (ML) [43] approach implemented in IQ-TREE v1.6.12 [44]. Nodal support was estimated combining the SH-like approximate likelihood ratio test (SH-aLRT) and the Ultrafast Bootstrap Approximation (UFboot) [45]. Redundant contigs with over $99 \%$ amino acid similarity were excluded. For those libraries containing viruses that were unlikely to be associated with Drosophila, taxonomic profiling and read mapping to components of the fly microbiome and diet were conducted using the CCMetagen software (default settings) [46] and BBMap v.37.98 (minid=0.95).

\section{Statistical analysis}

The assumption of data normality was assessed by visual inspection and using Kolmogorov-Smirnov (K-S) and Shapiro-Wilk's tests. As the data was not normally distributed, a Mann-Whitney-Wilcoxon test was used to compare the RNA virome composition with respect to the presence/ absence of members of the genus Wolbachia. Comparisons were made using raw and log-transformed data corresponding to RPM values (i.e. viral abundance) for each library. All analyses were performed using $\mathrm{R}$ software package rstatix (available at https://rpkgs.datanovia.com/rstatix/).

\section{RESULTS}

A total of 272 female flies were wild-caught in the Perth Hills, Western Australia and tested for Wolbachia infection through their F1s. The overall prevalence of members of the genus Wolbachia was $63.6 \%$ (173 out of 272), with frequencies at the three sampled locations varying from $54.8 \%$ (Raeburn Orchard, $n=73$ ) to $63.8 \%$ (Roleystone, $n=130$ ) and $72.5 \%$ (Cannington, $n=69$ ). We randomly selected 16 flies from the Raeburn Orchard field females for individual sequencing and 


\section{Drosophila simulans}

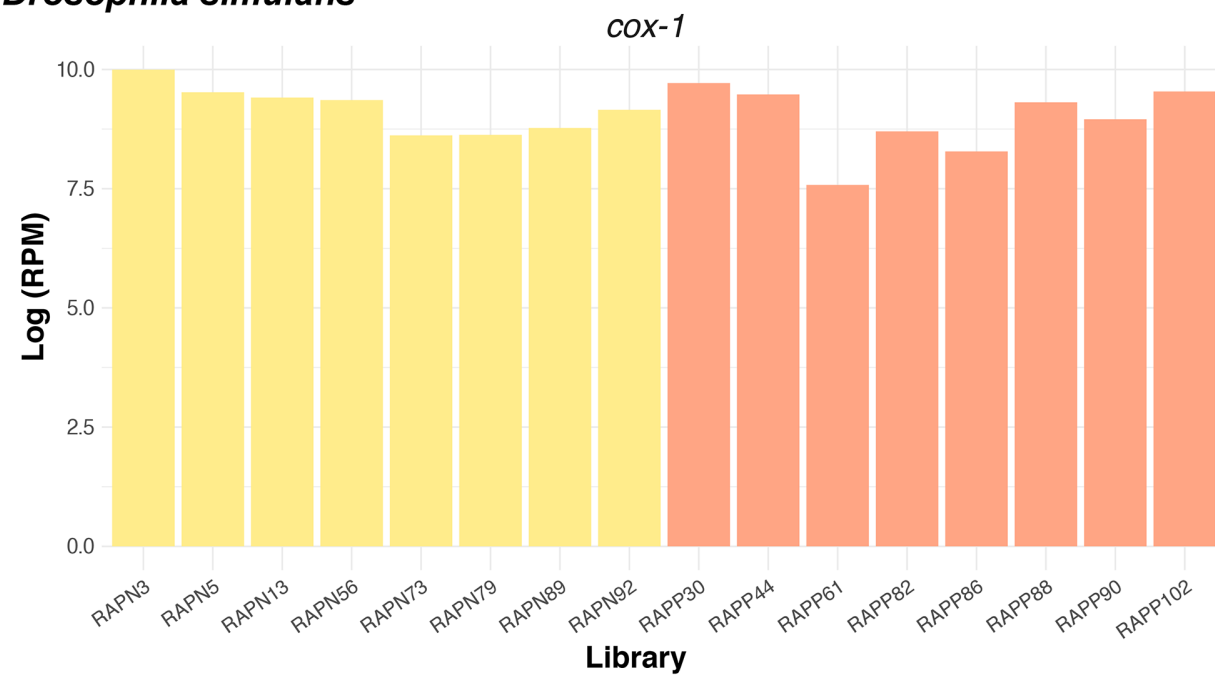

rp/32

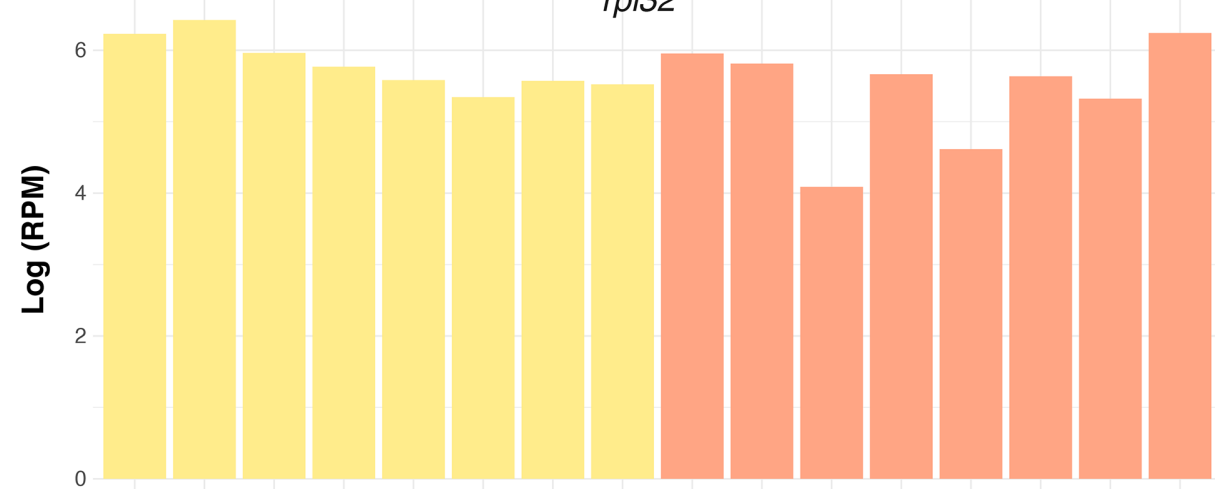

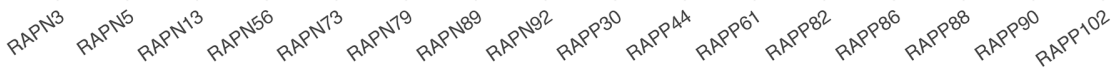

Library

\section{Wolbachia (wAu)}

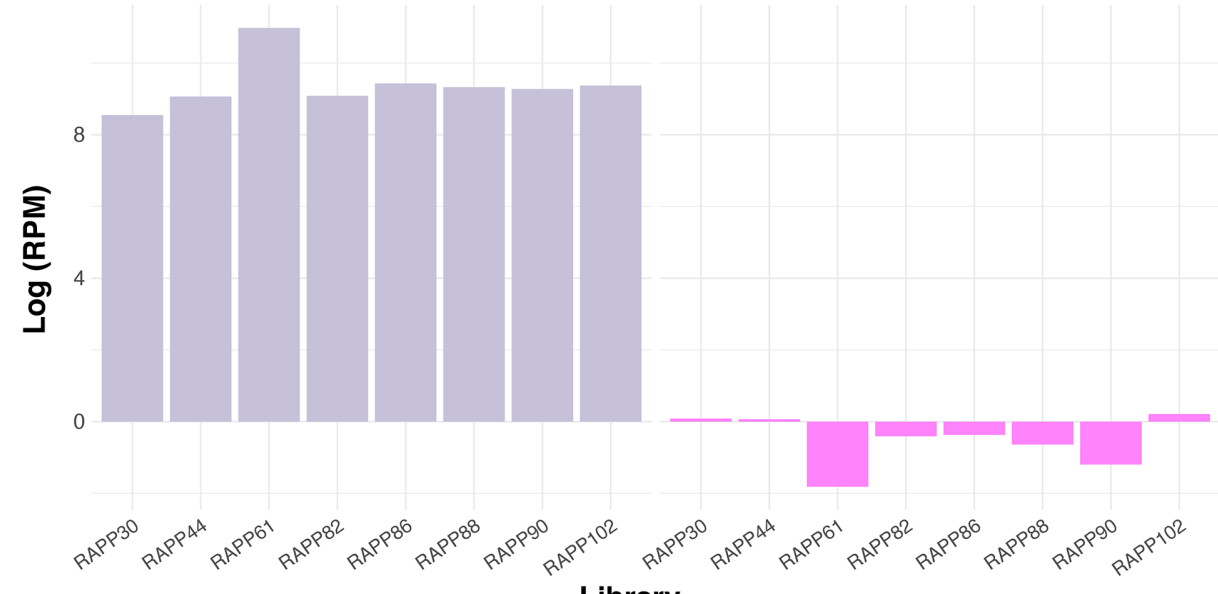

\section{Library}

Fig. 1. Comparison of the abundance levels of reference genes in Wolbachia-positive and Wolbachia-negative individual D. simulans (rpl32 and cox-1) and species of the genus Wolbachia (16S and cox1). 


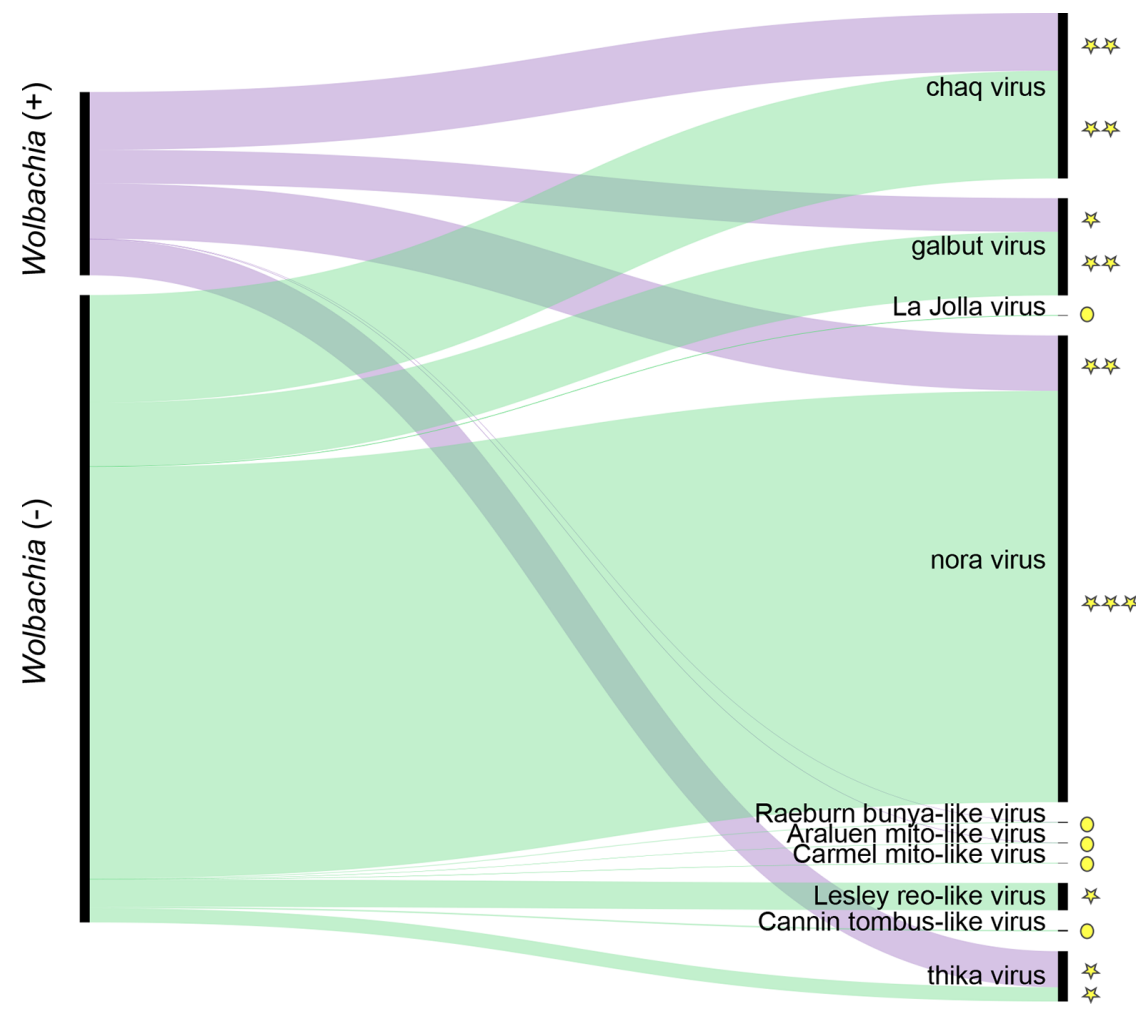

\section{Total RPM/virus}

iz $250,000-280,000$

is $30,000-80,000$

is $9,000-25,000$

$0<1,000$

Fig. 2. Comparison of viruses found in Wolbachia-positive and Wolbachia-negative $D$. simulans. The thickness of links is proportional to the total abundance (RPM) of each virus across the samples studied. The range of RPM values are represented with stars and circles.

RNA virus screening, representing eight Wolbachia-positive and eight Wolbachia-negative specimens.

We identified the Wolbachia strain in D. simulans using sequence-specific primers targeting the wsp gene. We further confirmed the occurrence of Wolbachia by mapping the reads back to the $w \mathrm{Ri}$ and $w \mathrm{Au} w s p$ genes. Most of the Wolbachiainfected flies showed a median coverage $>100$ reads, number of mapping reads $>40$, and coverage percentage $>90 \%$ to the reference $w \mathrm{Au}$ strain, confirming that infected flies harbour $w \mathrm{Au}$ rather than $w \mathrm{Ri}$. No reads mapped to the $w s p$ gene for library RAPP88 (Table S1, available in the online version of this article) despite the positive infection status determined using a Wolbachia-specific qPCR assay.

For comparison of virus diversity among libraries we mapped the reads of each library to stably expressed genes: $16 S$ and cox 1 in Wolbachia and rpl32 and cox 1 in D. simulans. This provided an internal control to identify any effect on viral abundance due to potential biases introduced during RNA extraction or library preparation. Although, as expected, there was moderate variation in the abundance values, expression levels of reference maker genes were relatively stable across libraries in both Wolbachia and D. simulans (Fig. 1).

Overall, we detected nine viruses in the 16 individual $D$. simulans studied here, five of which were novel (Fig. 2). Specifically, four viruses shared high sequence identity at the amino acid level $\left(>96 \%\right.$, e-value $\left.=0.00 \mathrm{E}^{+00}-4.2 \mathrm{E}^{-41}\right)$ to the $\mathrm{RdRp}$ of known RNA viruses, whereas the newly discovered viruses shared only between 32.6 and $62.6 \%$ amino acid identity to the best viral hit (e-value $\left.=0.00 \mathrm{E}^{+00}-1.4 \mathrm{E}^{-06}\right)($ Tables 1 , and S4). Similarly, phylogenetic analysis of the known virus sequences identified revealed close relationships with known Drosophilaassociated viruses: galbut virus (Partitiviridae), La Jolla virus (Iflaviridae), thika virus (Picornaviridae) and nora virus (Picornaviridae) (Fig. 3). In addition, we identified contigs related to 'chaq virus-like' sequences (>85\% amino acid sequence similarity). The novel viruses identified, that did not share close phylogenetic relationships to known viruses, were: Raeburn bunya-like virus (Bunyaviridae), Araluen mito-like virus (Mitoviridae), Carmel mito-like virus (Mitoviridae), Lesley reo-like virus (Reoviridae), and Cannin tombus-like virus (Tombusviridae) (Fig. 3). Similarity searches against the NCBI/nr database revealed that individual flies carried multiple invertebrate-associated viruses from different virus families. For example, up to six viruses were observed in a single $w$ Au-negative library (RAPN56) (Fig. 4, Table S2).

Some of the newly discovered RNA viruses identified here were probably infecting hosts other than $D$. simulans, and hence might be associated with the fly diet or microbiome. Specifically, these viruses were closely related to Phytomonas sp. TCC231 leishbunyavirus 1 (in the case of Raeburn bunyalike virus), Leptomonas pyrrhocoris RNA virus (Cannin tombus-like virus) and two mito-like viruses (Araluen 
Table 1. Summary of sequence similarity searches for viruses against the NCBI non-redundant database. Viral sequences listed below correspond to those included in phylogenetic analyses

\begin{tabular}{|c|c|c|c|c|c|c|}
\hline Query sequence & Library & $\begin{array}{l}\text { Wolbachia } \\
\text { infection }\end{array}$ & Length (nt) & Best match against the $\mathrm{NCBI} / \mathrm{nr}$ database & Similarity & e-value \\
\hline k119_3301_len12366_nora virus & RAPP86 & + & 12366 & AWY11063.1 putative replicase [nora virus] & 98.7 & $0.00 \mathrm{E}^{+00}$ \\
\hline k119_20553_len9231_thika virus & RAPP86 & + & 9231 & YP_009140561.1 putative polyprotein [thika virus] & 96.2 & $0.00 \mathrm{E}^{+00}$ \\
\hline k119_5914_len9220_thika virus & RAPN73 & - & 9220 & YP_009140561.1 putative polyprotein [thika virus] & 97.1 & $0.00 \mathrm{E}^{+00}$ \\
\hline k119_2329_len2049_Cannin tombus-like virus & RAPP88 & + & 2049 & $\begin{array}{l}\text { ASN64759.1 putative RNA-dependent RNA polymerase, } \\
\text { partial [Leptomonas pyrrhocoris RNA virus] }\end{array}$ & 48.4 & $3.80 \mathrm{E}^{-95}$ \\
\hline k119_4103_len1899_galbut virus & RAPN73 & - & 1899 & $\begin{array}{l}\text { AWY11176.1 putative RNA-dependent RNA polymerase } \\
\text { [galbut virus] }\end{array}$ & 96.7 & $0.00 \mathrm{E}^{+00}$ \\
\hline k119_273_len2671_Araluen mito-like virus & RAPN5 & - & 2671 & $\begin{array}{l}\text { QDH87474.1 RNA-dependent RNA polymerase, partial } \\
\text { [Mitovirus sp.] }\end{array}$ & 40.3 & $8.0 \mathrm{E}^{-96}$ \\
\hline k119_22084_len2612_Araluen mito-like virus & RAPN5 & - & 2612 & $\begin{array}{l}\text { QDH87474.1 RNA-dependent RNA polymerase, partial } \\
\text { [Mitovirus sp.] }\end{array}$ & 43.2 & $2.3 \mathrm{E}^{-103}$ \\
\hline k119_14037_len2615_Araluen mito-like virus & RAPN56 & - & 2615 & $\begin{array}{l}\text { QDH87474.1 RNA-dependent RNA polymerase, partial } \\
\text { [Mitovirus sp.] }\end{array}$ & 41.7 & $1.7 \mathrm{E}^{-98}$ \\
\hline k119_14318_len2822_Araluen mito-like virus & RAPN56 & - & 2822 & $\begin{array}{l}\text { QDH87474.1 RNA-dependent RNA polymerase, partial } \\
\text { [Mitovirus sp.] }\end{array}$ & 38.1 & $9.7 \mathrm{E}^{-92}$ \\
\hline
\end{tabular}

mito-like virus and Carmel mito-like virus) (Fig. 3, Table S3), that are associated with trypanosomatid protozoans and fungal hosts, respectively. In addition, taxonomic composition analyses as well as read mapping to common components of Drosophila diet or microbiome revealed that 0.1 and $0.05 \%$ of all non-rRNA reads mapped to fungi and trypanosomatids (cox1 gene marker), respectively. Hence, multiple microorganisms were present within individual fly libraries which may explain the occurrence of viruses not directly associated with Drosophila (files available at https://doi.org/10.6084/m9. figshare.c.5466690). In contrast, Lesley reo-like virus is likely to be a bona fide arthropod virus since it grouped with viruses previously detected in odonates and mosquitoes. In addition, it exhibited only approximately $24 \%$ nucleotide similarity with those reoviruses previously reported to be contaminants in Drosophila cell culture [25]. This indicates that Lesley reo-like virus is not a component of known contaminants and more is likely to be part of the natural $D$. simulans virome. The five newly identified viruses in this study corresponded to full or nearly complete genomes (see below). However, for the majority of the known viruses of Drosophila we only were able to identify ORFs encoding the RdRp: the exceptions were La Jolla virus and thika virus, for which we also predicted structural components corresponding to coat and capsid proteins.

We next characterized the virome profile present in D. simulans in relation to the $w \mathrm{Au}$ infection status (Fig. 2, Tables 1 and S4). Accordingly, we identified a slightly higher number $(n=9)$ of viruses in Wolbachia-negative flies compared with Wolbachia-positive flies $(n=6)$. Among these, galbut virus, nora virus, thika virus, as well as three novel viruses identified in this study - Raeburn bunya-like virus, Araluen mito-like virus and Cannin tombus-like virus - were present in $D$. simulans regardless of Wolbachia infection. Likewise, 'chaq virus-like' sequences were observed co-occurring with galbut virus in the two groups of $D$. simulans. In contrast, La Jolla virus, as well as the novel Carmel mito-like virus and Lesley reo-like virus, were only found in $w$ Au-negative flies. Overall, assembled viral contigs displayed high sequence similarity at the nucleotide and amino acid level within and between libraries and regardless of the presence or absence of members of the genus Wolbachia (Table S3).

We also assessed the potential effect of infection with members of the genus Wolbachia on the abundance of RNA viruses present in $w \mathrm{Au}$-infected and $w \mathrm{Au}$-uninfected flies. Overall, the number of non-rRNA reads represented approximately $50 \%$ of the total number of reads ( $n=743389696$ pairend reads) (Fig. S1). Furthermore, the RPM values among viruses infecting Wolbachia-negative and -positive infected flies was highly heterogeneous, ranging from 47 to 232346 and 5 to 37688 virus RPM, respectively. With the exception of thika virus, viruses present in both $w \mathrm{Au}$-positive and $w \mathrm{Au}$-negative flies were 1.87-40.17-fold more abundant in 
(a)

\section{Picornavirales}

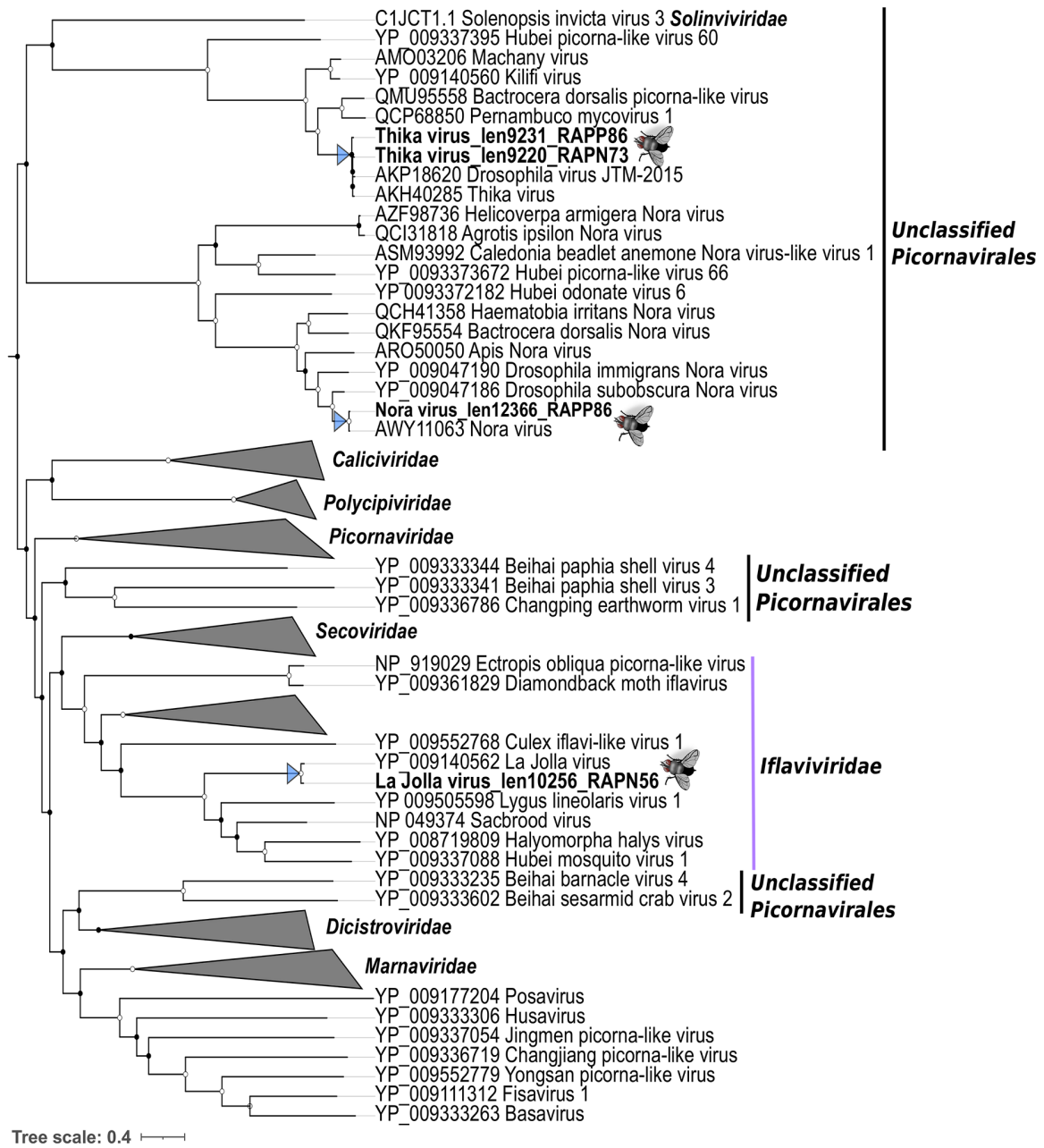

Tree scale: $0.4 \longmapsto$

AOR51385 Chaq virus-like 2

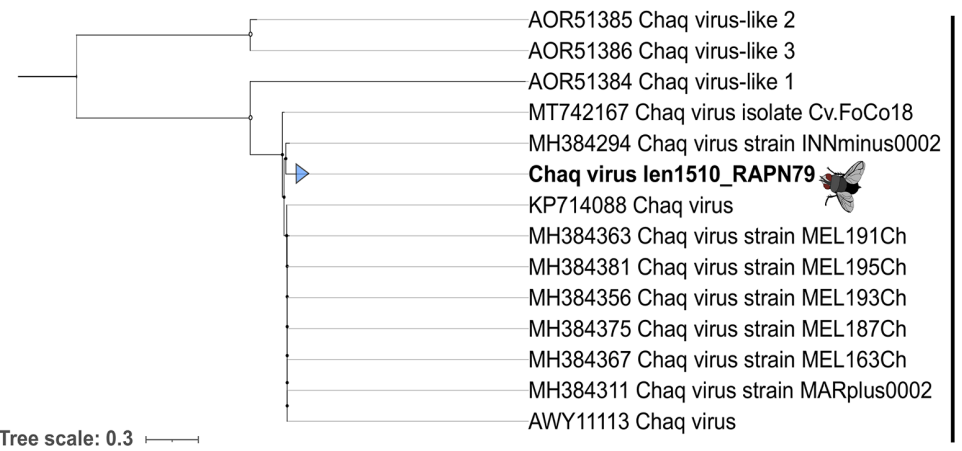

Unclassified

Fig. 3. Maximum likelihood phylogenetic trees of the viruses and virus-like sequences identified from $D$. simulans. The phylogenies were inferred based on the amino acid sequences of the RdRp of six virus taxonomic groups, whereas for chaq virus-like sequences we used a protein of unknown function. Virus family trees were rooted with relevant outgroups that are indicated with grey tips. Order-level trees and the chaq virus phylogeny (for which no suitable outgroup existed) were midpoint rooted. Coloured arrow tips represent probable (a, b) Drosophila-associated viruses and (c) non-Drosophila-associated viruses (i.e. those that were more probably associated with a component of fly diet or microbiome). Nodal support values greater than $80 \%$ (SH-aLRT) and $95 \%$ (UFboot) are indicated with white circular shapes at the nodes. Branch lengths are projected using scale bars below each tree. 
Fig. 3. (Cont.)
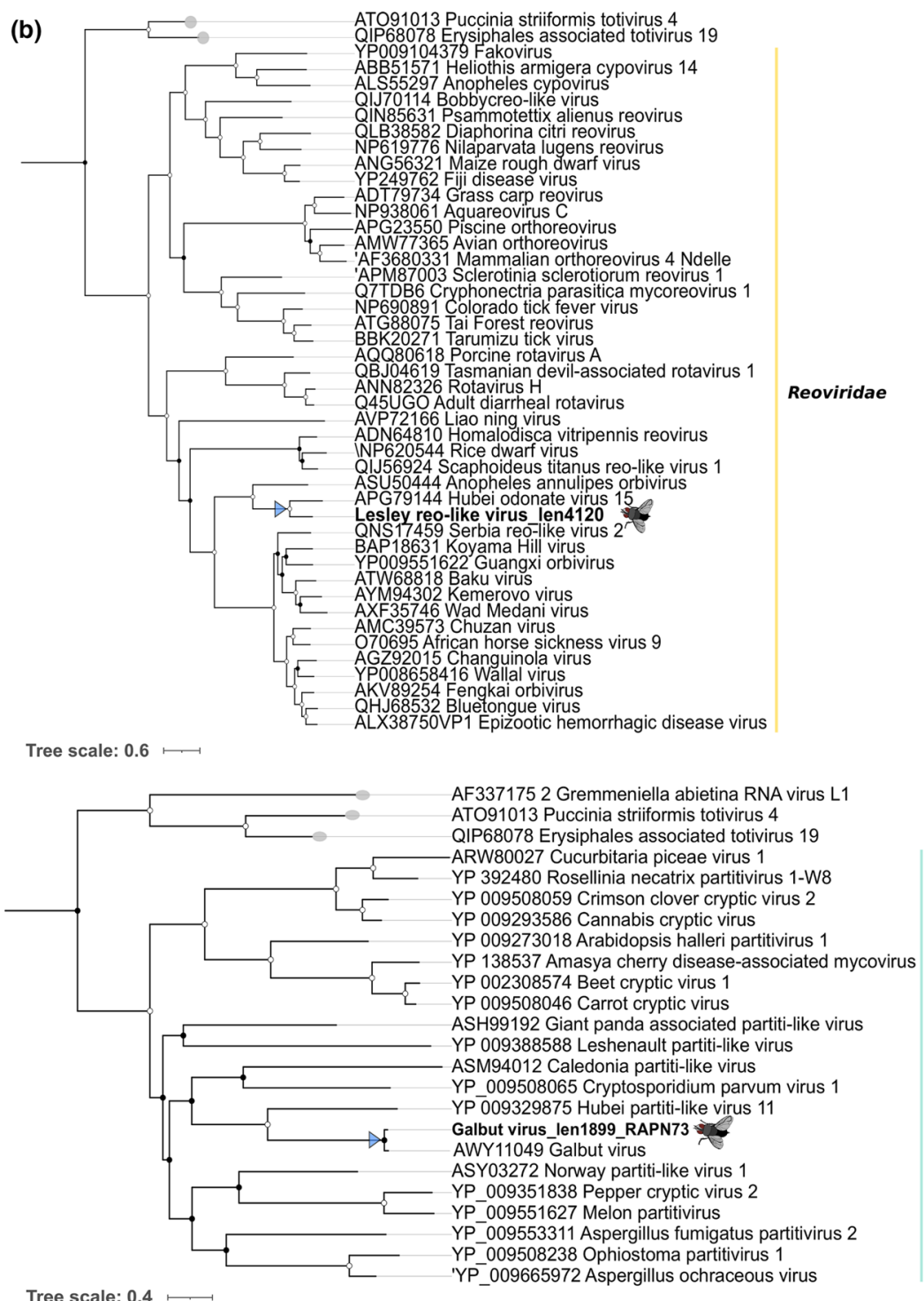

Partitiviridae 
Fig. 3. (Cont.)

(c)
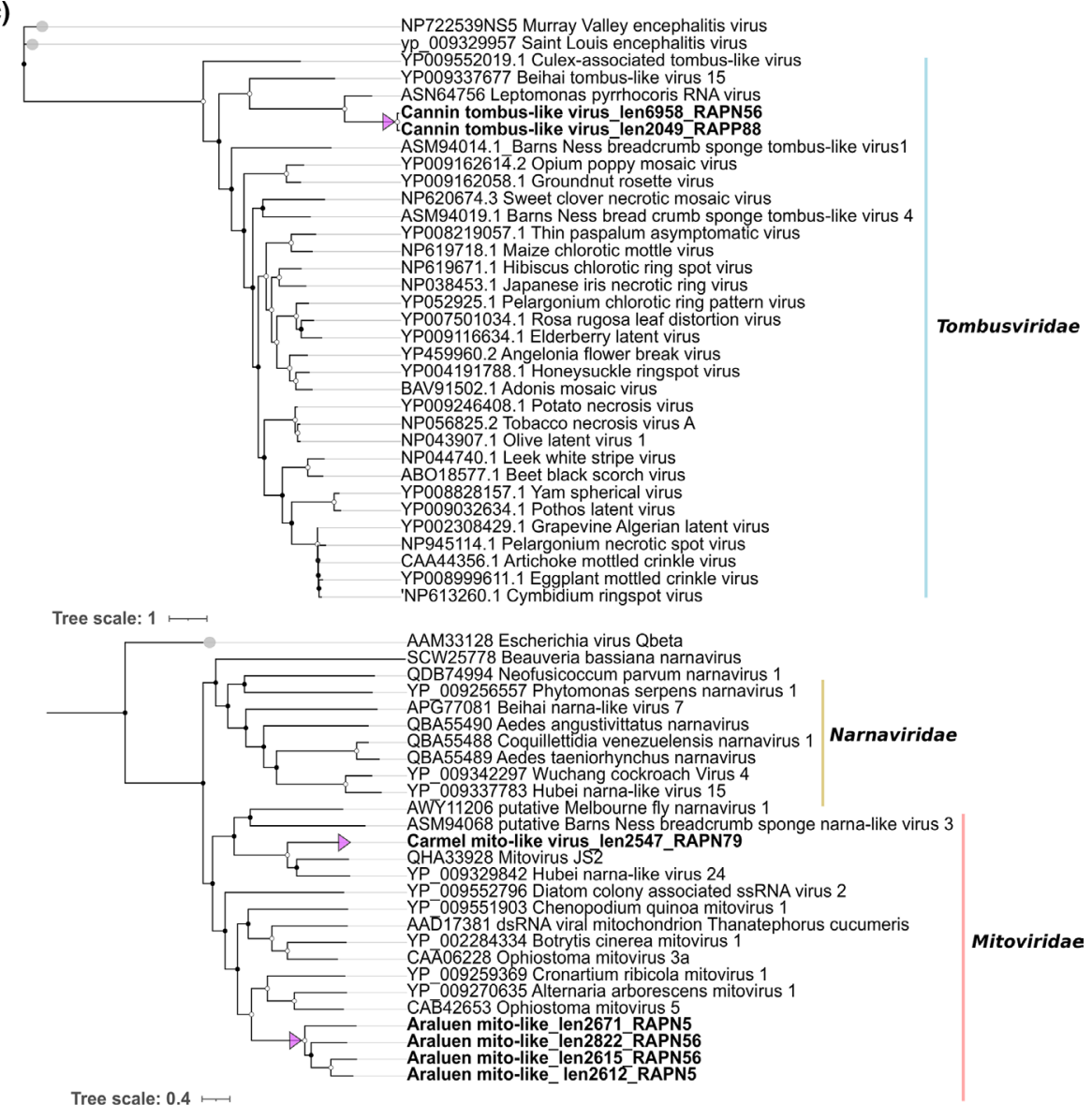

Bunyavirales

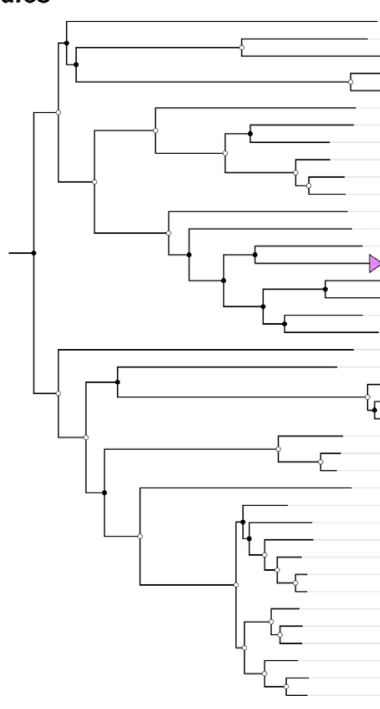

QJD26146 Schistocephalus solidus bunya-like virus AR
009300680 Shayang Spider Virus 1 AIT7347 Lassa mammarenavirus Arenaviridae AlK25571 Gairo mammarenavirus Arenavirida YP 008003507 Lone Star virus Phenuiviridae ABD51514 Rift Valley fever virus QIJ 50048 partial Boyle bunya-like virus 作 a AYD61653 Blechmonás ayalai Teishbunyavirus 1 ANJ59513 Leptomonas moramango leishbunyavirus

YP-0006362282 Maporal virus

\begin{tabular}{l|l} 
YP-00944945 C Calla lily chllorotic spot virus & Toposviridae \\
YP- 009345145 Pepper chlorotic spot virus & Top
\end{tabular}

AVM 187194 Hainan bunya-like virus

YP-009507885 Koongol virus

YP-009512925 Wyeomyia orthobunyavirus

$\mathrm{NP}-047211$ Bunyamwera virus
$\mathrm{YP}-00966865$ Tensaw virus

YP-008400138 Brazoran virus

YP-009362071 Madrid virus

$\mathrm{NP}^{-} 982304 \mathrm{RNA}$ Oropouche virus

YP-009666911 Schmallenberg virus
YP_-006590082 Simbu orthobunyavirus

Peribunyaviridae 


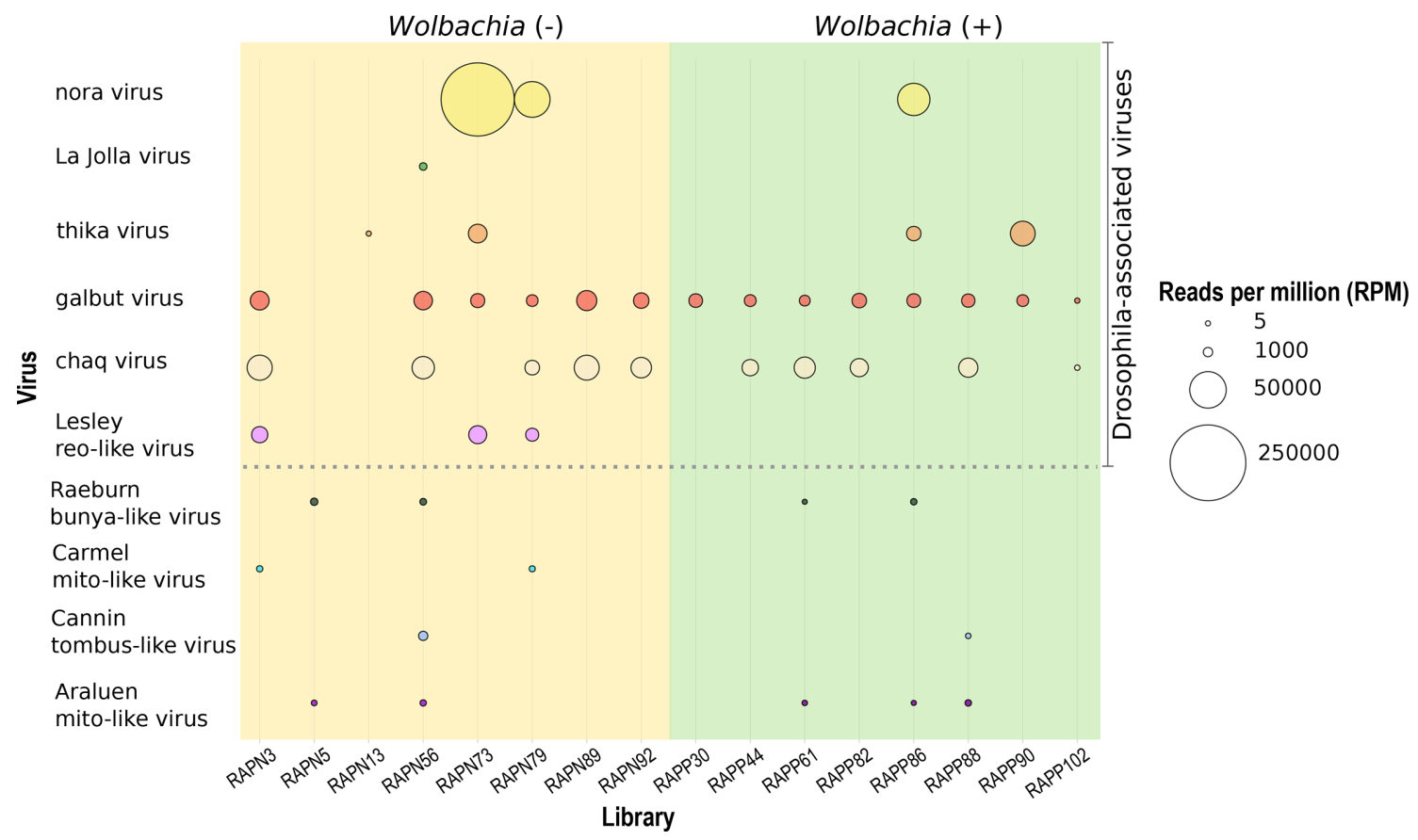

Fig. 4. Representation of virome composition and abundance (RPM) across Wolbachia-positive and -negative libraries. Each library represents an individual $D$. simulans fly. All reads likely to be due to index-hopping have been excluded.

the $w \mathrm{Au}$-negative individuals than $w \mathrm{Au}$-positive $D$. simulans. In contrast, the abundance of thika virus was 0.39 -fold higher in the Wolbachia-positive flies (Fig. 3, Table S2). However, despite this variation in virus abundance levels between groups, there was a non-significant difference between $w \mathrm{Au}-$ negative and $w \mathrm{Au}$-positive $D$. simulans (Mann-WhitneyWilcoxon test; Fig. 5). In the case of the viruses only detected in the $w \mathrm{Au}$-negative flies, La Jolla virus was present in a single library in moderate abundance $(\mathrm{RPM}=378)$, whilst the newly discovered Lesley reo-like virus was detected in four out of eight libraries (RPM=3360-8749) (Table S2). Although an interesting result, the limited sample size $(n=16)$ means that these observations should be taken with caution and that larger sample sizes are needed for corroboration.

\section{DISCUSSION}

The occurrence and spread of Wolbachia infection has been widely documented in natural populations of Drosophila $[10,30,47]$. Indeed, $D$. simulans is commonly used as an experimental model to investigate the interactions within the tripartite Drosophila-Wolbachia-virus system. In Australia, D. simulans can be naturally infected with two strains of Wolbachia from supergroup A $w \mathrm{Au}$ and $w \mathrm{Ri}$. While $w \mathrm{Ri}$ has been gradually displacing $w A u$ in eastern Australia, reflected in the changing infection frequencies in surveyed populations since 2004, D. simulans from the west coast of Australia only harbour $w \mathrm{Au}[30]$. A simple and plausible explanation for this difference is the geographic separation of $D$. simulans populations inhabiting the east and west coasts of Australia and the challenging environmental conditions posed by the intervening desert [30].

We corroborated the presence of Wolbachia infection across samples by identifying the $w s p, 16 S$ and $\operatorname{cox} 1$ marker genes. The lack of reads mapping to the library RAPP88 might reflect either low levels of $w s p$ RNA molecules present in the input for library preparation or high variability compared with the reference sequence. Although Wolbachia density was not experimentally assessed, the similar levels of $16 \mathrm{~S}$ and cox-1 abundance across libraries indicate no appreciable biases in the library preparation and RNA sequencing steps.

Estimates from previous surveys indicated that the frequency of the $w$ Au strain in Western Australia exceeded $50 \%$ in $D$. simulans [30]. This is consistent with the data provided here and indicates that Wolbachia might be present in a significant proportion of the natural fly population, at least around Perth. Although $w \mathrm{Au}$ does not cause cytoplasmic incompatibility, its spread is suggested to confer fitness advantages (increased survival and/or reproduction) to the host, including antiviral protection $[48,49]$, that might favour its spread and prevent the bacteria from being eliminated from $D$. simulans populations $[30,50]$. However, our comparison of Wolbachia-infected and -uninfected D. simulans in western Australia revealed no clear effect of Wolbachia infection on virome composition and viral abundance between Wolbachia infected and uninfected animals. Although our analysis is based on a small sample of individual flies, the apparent absence of a Wolbachia-mediated virus protection effect in natural $D$. simulans is compatible with previous findings on $D$. melanogaster naturally infected 


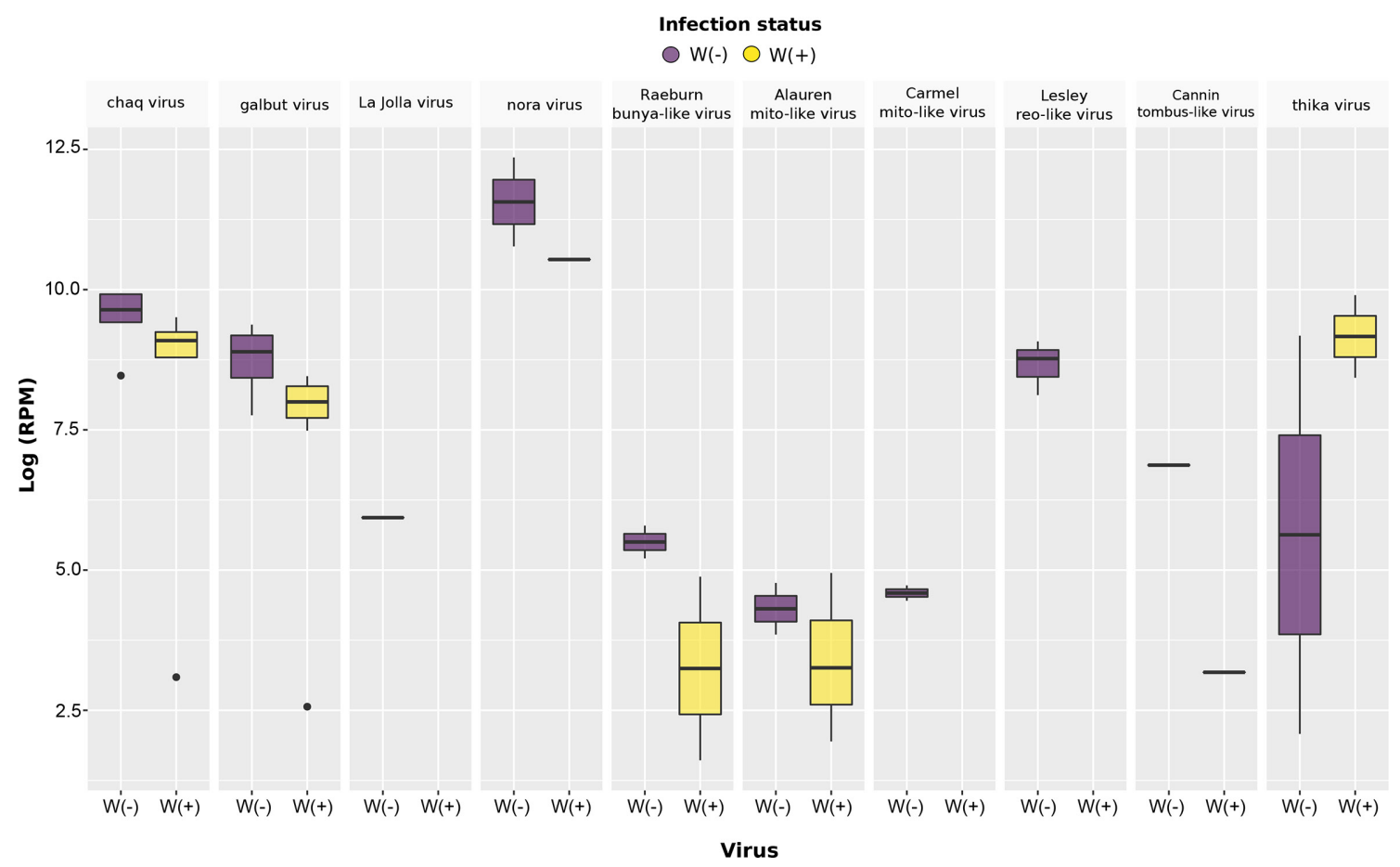

Fig. 5. Abundance distribution of nine RNA viruses and the chaq virus-like sequences identified across individual Wolbachia-positive and Wolbachia-negative D. simulans. A non-significant difference was observed between Wolbachia-infected and uninfected flies using the Mann-Whitney U test.

with $w$ Mel in eastern Australia [27], in which virus protection was not observed regardless of the Wolbachia infection status and Wolbachia density. Even so, the absence of a significant association between $w \mathrm{Au}$ infection and virus diversity does not necessarily translate into a homogeneous effect of $w \mathrm{Au}$ on the different viruses identified here. For example, it is plausible that the restricted presence of La Jolla virus and the newly identified Lesley reo-like virus in Wolbachia-free flies could reflect some effect of antiviral protection in D. simulans $[27,51]$. Indeed, contrasting results were observed in D. melanogaster, where La Jolla virus was widely distributed across different libraries [27]. Although this might provide insights into $w \mathrm{Au}$-virus interactions, studies based on larger sample sizes are clearly needed to determine whether the apparent association between La Jolla virus and Wolbachia-uninfected flies observed here is an artefact due to small sample sizes. Indeed, it is notable that La Jolla virus was so rarely detected in the D. simulans flies studied here.

It has previously been shown that the $w A u$ strain of Wolbachia has a protective role against virus infection in D. simulans when flies are challenged with FHV and DCV in a laboratory setting $[24,32]$. Moreover, the $w A u$ strain is protective against the dengue (DENV) and zika (ZIKV) viruses in Aedes aegypti mosquitoes [52]. Although our observation of an apparent lack of Wolbachia-mediated antiviral protection contrasts with those obtained previously, it is likely that differences may depend on Wolbachiahost species combinations and natural or artificial viral infections, which may also explain the contrasting results for La Jolla virus. Indeed, most of the available studies have documented the antiviral effect in transinfected insect hosts with non-natural strains of Wolbachia and viruses under laboratory conditions, as opposed to the study of the natural virome undertaken here.

It is noteworthy that ecological variables such as temperature might affect Wolbachia-virus-host interactions. Here, we collected flies during the Western Australian winter (mean temperature of $21^{\circ} \mathrm{C}$ daylight time) and the specimens were maintained at $19^{\circ} \mathrm{C}$ under laboratory conditions. Lower temperatures have been associated with an increase in viral resistance against DCV in D. melanogaster infected with $w \mathrm{Mel}$ and $w \mathrm{MelCS}$ [53]. Similarly, variations in host developmental temperature have been associated with differences in Wolbachia-mediated virus blocking in natural populations [53]. In this context, flies developed at lower temperature $\left(18^{\circ} \mathrm{C}\right)$ exhibited a reduction in Wolbachia-conferred antiviral protection. On the other hand, the presence of Wolbachia has been suggested to influence host temperature preferences. For instance, $w \mathrm{Ri}$ and $w$ Ho strains seem to manipulate $D$. simulans flies to seek cooler temperatures [54]. Although the effect of temperature on $w \mathrm{Au}$ and $D$. simulans need to be tested, this indicates that the results observed here as well as a protective scenario might be temperature-dependent. This highlights the importance of careful future studies of the interactions within the host-virus-Wolbachia system along with environmental factors in natural populations [55-57]. 
As well as the small sample size, an important caveat of our work is that we explored the Wolbachia-mediated virus protection in terms of virus abundance levels reflected in RPM values. This provides insights into virus resistance, but not into tolerance or host survival. Thus, it is still possible that Wolbachia is increasing tolerance to virus infection as has been documented for DCV [32]. In addition, although we were not able to assess Wolbachia density, results of previous studies have indicated that $w \mathrm{Au}$ is maintained at high-density in D. simulans and has a role in virus blocking [58]. Further research is clearly needed to assess these features in natural populations to determine any link with antiviral protection.

Collectively, comparisons of the virome composition in $w A u$ infected and uninfected $D$. simulans revealed the presence of natural and relatively highly abundant Drosophila-associated viruses in both groups [25, 27, 59]. Consistently with results from previous studies, we noted the co-occurrence of chaq virus-like sequences and galbut virus, supporting the idea that chaq virus might be part of a satellite-helper virus system or an additional segment associated with galbut virus $[27,60]$. In addition to insectassociated viruses, we identified viruses that are likely to infect other hosts and hence were likely to be associated with components of the diet or microbiome of $D$. simulans [61]. For instance, novel viruses from the families Tombusviridae and Bunyaviridae were related to viruses in trypanosomatid protozoa (Leptomonas and Leishmania). Similarly, given their normal host range distribution, the novel viruses from the family Narnaviridae might be associated with fungal hosts. Evidence of trypanosomatids and fungi in the gut of several species of Drosophila, with effects on larvae eclosion and pupation times $[61,62]$, has been reported. This, in turn, highlights the extent to which Australian D. simulans can be parasitized in nature [62-66].

In sum, we provide a preliminary framework for assessing the effect of the $w \mathrm{Au}$ strain on the virome of $D$. simulans, using a meta-transcriptomic analysis of individual $w \mathrm{Au}$-infected and uninfected flies. In doing so we identified Drosophila-associated viruses along with five novel viruses likely to be associated with fly diet or microbiome. Although our sample size is small, we saw no detectable Wolbachia-associated antiviral effect on virus composition and abundance, although the approach taken prevented us from drawing conclusions on virus tolerance. Further research employing larger sample sizes over broad spatial scales, including different WolbachiaDrosophila combinations, will enable a more nuanced understanding of Wolbachia-virus dynamics in wild Drosophila populations.

\section{Funding information}

This research was funded by an Australian Research Council Australian Laureate Fellowship to E.C.H (grant FL170100022), a National Health and Medical Research Council (NHMRC) Research Fellowship to A.A.H. (grant 1118640), and an NHMRC Project grant (grant GNT1103804).

\section{Acknowledgements}

We thank Vanessa White for research assistance support to conduct this study.

\section{Author contributions}

Conceptualization, M.S., A.A.H. and E.C.H.; methodology, A.S.O.-B., M.S. A.A.H. and E.C.H., formal analysis, A.S.O.-B.; investigation, A.S.O.-B. and M.S.; resources, A.A.H. and E.C.H.; writing - original draft preparation A.S.O.-B.; writing - review and editing A.A.H., M.S and E.C.H.; visualization, A.S.O.-B.; supervision, E.C.H.; All authors have read and agreed to the published version of the manuscript.

Conflicts of interest

The authors declare that there are no conflicts of interest.

References

1. Zug R, Hammerstein P. Bad guys turned nice? A critical assessment of Wolbachia mutualisms in arthropod hosts. Biol Rev Camb Philos Soc 2015:90:89-111.

2. Ross PA, Turelli M, Hoffmann AA. Evolutionary ecology of Wolbachia releases for disease control. Annu Rev Genet 2019;53:93-116.

3. Ros VID, Fleming VM, Feil EJ, Breeuwer JAJ. How diverse is the genus Wolbachia? Multiple-gene sequencing reveals a putatively new Wolbachia supergroup recovered from spider mites (Acari: Tetranychidae). Appl Environ Microbiol 2009;75:1036-1043.

4. Scholz M, Albanese D, Tuohy K, Donati C, Segata N, et al. Large scale genome reconstructions illuminate Wolbachia evolution. Nat Commun 2020;11:1-11

5. Zouache K, Voronin D, Tran-Van V, Mousson L, Failloux A-B, et al. Persistent Wolbachia and cultivable bacteria infection in the reproductive and somatic tissues of the mosquito vector Aedes albopictus. PLoS One 2009:4:e6388.

6. Dobson SL, Bourtzis K, Braig HR, Jones BF, Zhou W, et al. Wolbachia infections are distributed throughout insect somatic and germ line tissues. Insect Biochem Mol Biol 1999:29:153-160.

7. Frydman HM, Li JM, Robson DN, Wieschaus E. Somatic stem cell niche tropism in Wolbachia. Nature 2006:441:509-512.

8. Tsai K-H, Lien J-C, Huang C-G, Wu W-J, Chen W-J. Molecular (sub) grouping of endosymbiont Wolbachia infection among mosquitoes of Taiwan. J Med Entomol 2004:41:677-683.

9. Strunov A, Kiseleva E. Drosophila melanogaster brain invasion: Pathogenic Wolbachia in central nervous system of the fly. Insect Sci 2016;23:253-264

10. Turelli M, Cooper BS, Richardson KM, Ginsberg PS, Peckenpaugh B et al. Rapid global spread of wRi-like Wolbachia across multiple Drosophila. Curr Biol 2018;28:963-971.

11. O'Neill SL, Hoffmann A, Werren J. Influential Passengers: Inherited Microorganisms and Arthropod Reproduction. 1st ed. Oxford University Press, 1997.

12. Iturbe-Ormaetxe I, O'Neill SL. Wolbachia-host interactions: Connecting phenotype to genotype. In: Current Opinion in Microbiology, Vol. 10. Elsevier Current Trends, 2007. pp. 221-224.

13. Dedeine F, Vavre F, Fleury F, Loppin B, Hochberg ME, et al Removing symbiotic Wolbachia bacteria specifically inhibits oogenesis in a parasitic wasp. Proc Natl Acad Sci U S A 2001;98:6247-6252.

14. Hoerauf A, Nissen-Pähle $K$, Schmetz C, Henkle-Dührsen $K$, Blaxter ML, et al. Tetracycline therapy targets intracellular bacteria in the filarial nematode Litomosoides sigmodontis and results in filarial infertility. J Clin Invest 1999;103:11-18.

15. Nikoh N, Hosokawa T, Moriyama M, Oshima K, Hattori M, et al Evolutionary origin of insect-Wolbachia nutritional mutualism. Proc Natl Acad Sci U S A 2014;111:10257-10262.

16. Ju J-F, Bing X-L, Zhao D-S, Guo Y, Xi Z, et al. Wolbachia supplement biotin and riboflavin to enhance reproduction in planthoppers. ISME J 2020;14:676-687.

17. Hosokawa T, Koga R, Kikuchi Y, Meng XY, Fukatsu T. Wolbachia as a bacteriocyte-associated nutritional mutualist. Proc Natl Acad Sci U S A 2010;107:769-774. 
18. Tantowijoyo W, Andari B, Arguni E, Budiwati N, Nurhayati I, et al. Stable establishment of wMel Wolbachia in Aedes aegypti populations in Yogyakarta, Indonesia. Barker CM, editor. PLoS Negl Trop Dis 2020;14:e0008157.

19. Moreira LA, Iturbe-Ormaetxe I, Jeffery JA, Lu G, Pyke AT, et al. A Wolbachia symbiont in Aedes aegypti limits infection with Dengue, Chikungunya, and Plasmodium. Cell 2009:139:1268-1278.

20. Rancès $E, Y e$ YH, Woolfit M, McGraw EA, O'Neill SL. The relative importance of innate immune priming in Wolbachia-mediated dengue interference. PLoS Pathog 2012;8:e1002548.

21. Zug R, Hammerstein P. Wolbachia and the insect immune system: what reactive oxygen species can tell us about the mechanisms of Wolbachia-host interactions. Front Microbiol 2015;6:1201.

22. Terradas G, Joubert DA, McGraw EA. The RNAi pathway plays a small part in Wolbachia-mediated blocking of dengue virus in mosquito cells. Sci Rep 2017;7:43847.

23. Teixeira L, Ferreira A, Ashburner M. The bacterial symbiont Wolbachia induces resistance to RNA viral infections in Drosophila melanogaster. PLoS Biol 2008;6:e2.

24. Martinez J, Longdon B, Bauer S, Chan Y-S, Miller WJ, et al. Symbionts commonly provide broad spectrum resistance to viruses in insects: a comparative analysis of Wolbachia strains. PLoS Pathog 2014;10:e1004369.

25. Webster CL, Waldron FM, Robertson S, Crowson D, Ferrari G, et al. The discovery, distribution, and evolution of viruses associated with Drosophila melanogaster. PLoS Biol 2015;13:e1002210.

26. Hedges LM, Brownlie JC, O'Neill SL, Johnson KN. Wolbachia and virus protection in insects. Science 2008;322:702

27. Shi M, White VL, Schlub T, Eden J-S, Hoffmann AA, et al. No detectable effect of Wolbachia wMel on the prevalence and abundance of the RNA virome of Drosophila melanogaster. Proc Biol Sci 2018;285:20181165

28. Lachaise D, Cariou M-L, David JR, Lemeunier F, Tsacas L, et al. Historical biogeography of the Drosophila melanogaster species subgroup. In: Evolutionary Biology. Springer US, 1988. pp. 159-225.

29. Mather W. Additions to the Drosophila fauna of Australia. Univ Queensl Pap 1960;1.

30. Kriesner P, Hoffmann AA, Lee SF, Turelli M, Weeks AR. Rapid sequential spread of two Wolbachia variants in Drosophila simulans. Charlat S, editor. PLoS Pathog 2013;9:e1003607.

31. Parsons PA, Bock IR. The population biology of Australian Drosophila. Annu Rev Ecol Syst 1979;10:229-245.

32. Osborne SE, Leong YS, O'Neill SL, Johnson KN. Variation in antiviral protection mediated by different Wolbachia strains in Drosophila simulans. Schneider DS, editor. PLoS Pathog 2009:5:e1000656.

33. Casiraghi M, Bordenstein SR, Baldo L, Lo N, Beninati $T$, et al. Phylogeny of Wolbachia pipientis based on gltA, groEL and fts $Z$ gene sequences: Clustering of arthropod and nematode symbionts in the F supergroup, and evidence for further diversity in the Wolbachia tree. Microbiology (Reading) 2005;151:4015-4022.

34. Hoffmann AA, Clancy D, Duncan J. Naturally-occurring Wolbachia infection in Drosophila simulans that does not cause cytoplasmic incompatibility. Heredity (Edinb) 1996;76:1-8.

35. Endersby NM, Mckechnie SW, Vogel H, Gahan LJ, Baxter SW, et al. Microsatellites isolated from diamondback moth, Plutella xylostella (L.), for studies of dispersal in Australian populations. Mol Ecol Notes 2005:5:51-53

36. Bolger AM, Lohse M, Usadel B. Trimmomatic: a flexible trimmer for Illumina sequence data. Bioinformatics 2014:30:2114-2120.

37. Grabherr MG, Haas BJ, Yassour M, Levin JZ, Thompson DA, et al. Full-length transcriptome assembly from RNA-Seq data without a reference genome. Nat Biotechnol 2011;29:644-652.

38. Buchfink B, Xie C, Huson DH. Fast and sensitive protein alignment using DIAMOND. Nat Methods 2015;12:59-60.

39. Rice P, Longden L, Bleasby A. EMBOSS: The European Molecular Biology Open Software Suite. Trends Genet 2000;16:276-277.
40. Zdobnov EM, Apweiler R. InterProScan - an integration platform for the signature-recognition methods in InterPro. Bioinformatics 2001;17:847-848

41. Lay CL, Shi M, Buček A, Bourguignon T, Lo N, et al. Unmapped RNA virus diversity in termites and their symbionts. Viruses 2020;12:1145.

42. Katoh K, Standley DM. MAFFT Multiple Sequence Alignment Software Version 7: Improvements in Performance and Usability. Mol Biol Evol 2013;30:772-780.

43. Felsenstein J. Evolutionary trees from DNA sequences: A maximum likelihood approach. J Mol Evol 1981;17:368-376.

44. Nguyen L-T, Schmidt HA, von Haeseler A, Minh BQ. IQ-TREE: A fast and effective stochastic algorithm for estimating maximumlikelihood phylogenies. Mol Biol Evol 2015;32:268-274.

45. Hoang DT, Chernomor O, Von Haeseler A, Minh BQ, Vinh LS UFBoot2: Improving the ultrafast bootstrap approximation. Mol Biol Evol 2018;35:518-522.

46. Marcelino VR, Clausen P, Buchmann JP, Wille M, Iredell JR, et al. CCMetagen: comprehensive and accurate identification of eukaryotes and prokaryotes in metagenomic data. bioRxiv 2019;641332.

47. Turelli M, Hoffmann AA. Rapid spread of an inherited incompatibility factor in California Drosophila. Nature 1991;353:440-442.

48. Ogunlade ST, Adekunle AI, Meehan MT, Rojas DP, McBryde ES. Modeling the potential of wAu-Wolbachia strain invasion in mosquitoes to control Aedes-borne arboviral infections. Sci Rep 2020;10:16812.

49. Mancini MV, Herd CS, Ant TH, Murdochy SM, Sinkins SP Wolbachia strain wAu efficiently blocks arbovirus transmission in Aedes albopictus. Samy AM, editor. PLoS Negl Trop Dis 2020;14:e0007926.

50. Correa CC, Ballard JWO. Wolbachia associations with insects: Winning or losing against a master manipulator. Front Ecol Evol 2016:3:153.

51. Habayeb MS, Cantera R, Casanova G, Ekström J-0, Albright S, et al. The Drosophila Nora virus is an enteric virus, transmitted via feces. J Invertebr Pathol 2009:101:29-33.

52. Ant TH, Herd CS, Geoghegan V, Hoffmann AA, Sinkins SP. The Wolbachia strain wAu provides highly efficient virus transmission blocking in Aedes aegypti. Schneider DS, editor. PLOS Pathog 2018;14:e1006815.

53. Chrostek E, Martins N, Marialva MS, Teixeira L. Wolbachiaconferred antiviral protection is determined by developmental temperature. bioRxiv 2020;2020.06.24.169169.

54. Hague MTJ, Caldwell CN, Cooper BS. Pervasive effects of Wolbachia on host temperature preference. MBio 2020;11:1-15.

55. Johnson KN. The impact of Wolbachia on virus infection in mosquitoes. Viruses 2015;7:5705-5717

56. Cao L-J, Jiang W, Hoffmann AA. Life history effects linked to an advantage for wAu Wolbachia in Drosophila. Insects 2019;10:126.

57. Fenton A, Johnson KN, Brownlie JC, Hurst GDD. Solving the Wolbachia paradox: Modeling the tripartite interaction between host, Wolbachia, and a natural enemy. Am Nat 2011;178:333-342.

58. Osborne SE, Iturbe-Ormaetxe I, Brownlie JC, O'Neill SL, Johnson KN. Antiviral protection and the importance of Wolbachia density and tissue tropism in Drosophila simulans. Appl Environ Microbiol 2012;78:6922-6929.

59. Palmer WH, Medd NC, Beard PM, Obbard DJ. Isolation of a natural DNA virus of Drosophila melanogaster, and characterisation of host resistance and immune responses. PLOS Pathog 2018;14:e1007050.

60. Cross ST, Maertens BL, Dunham TJ, Rodgers CP, Brehm AL, et al. Partitiviruses infecting Drosophila melanogaster and Aedes aegypti exhibit efficient biparental vertical transmission. J Virol 2020;94.

61. Ebbert MA, Marlowe JL, Burkholder JJ. Protozoan and intracellular fungal gut endosymbionts in Drosophila: prevalence and fitness effects of single and dual infections. J Invertebr Pathol 2003;83:37-45. 
62. Wilfert L, Longdon B, Ferreira AGA, Bayer F, Jiggins FM. Trypanosomatids are common and diverse parasites of Drosophila. Parasitology 2011;138:858-865.

63. Chandler JA, James PM. Discovery of trypanosomatid parasites in globally distributed Drosophila species. Braga ÉM, editor. PLoS One 2013;8:e61937.

64. Ebbert MA, Burkholder JJ, Marlowe JL. Trypanosomatid prevalence and host habitat choice in woodland Drosophila. J Invertebr Pathol 2001;77:27-32
65. Lemaitre B, Nicolas E, Michaut L, Reichhart JM, Hoffmann JA The dorsoventral regulatory gene cassette spatzle/Toll/Cactus controls the potent antifungal response in Drosophila adults. Cell 1996;86:973-983.

66. Naranjo-Lázaro JM, Mellín-Rosas MA, González-Padilla VD, Sánchez-González JA, Moreno-Carrillo G, et al. Susceptibility of Drosophila suzukii Matsumura (Diptera: Drosophilidae) to entomophatogenic fungi. Southwestern Entomologist 2014;39:201-203.

Five reasons to publish your next article with a Microbiology Society journal

1. The Microbiology Society is a not-for-profit organization.

2. We offer fast and rigorous peer review - average time to first decision is 4-6 weeks.

3. Our journals have a global readership with subscriptions held in research institutions around the world.

4. $80 \%$ of our authors rate our submission process as 'excellent' or 'very good'.

5. Your article will be published on an interactive journal platform with advanced metrics

Find out more and submit your article at microbiologyresearch.org. 


\section{University Library}

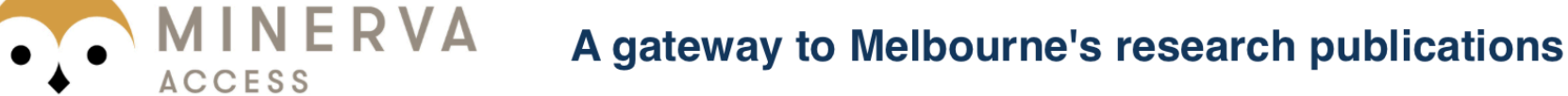

Minerva Access is the Institutional Repository of The University of Melbourne

Author/s:

Ortiz-Baez, AS;Shi, M;Hoffmann, AA;Holmes, EC

Title:

RNA virome diversity and Wolbachia infection in individual Drosophila simulans flies

Date:

2021-01-01

Citation:

Ortiz-Baez, A. S., Shi, M., Hoffmann, A. A. \& Holmes, E. C. (2021). RNA virome diversity and Wolbachia infection in individual Drosophila simulans flies. JOURNAL OF GENERAL VIROLOGY, 102 (10), https://doi.org/10.1099/jgv.0.001639.

Persistent Link:

http://hdl.handle.net/11343/296534

License:

CC BY 